\title{
High humidity aggravates the severity of arthritis in collagen-induced arthritis mice by upregulating xylitol and L-pyroglutamic acid
}

\author{
Mingzhu Wang ${ }^{\dagger}$, Jiao Chen ${ }^{\dagger}$, Xiaoying Lin, Lin Huang, Haichang Li, Chengping Wen ${ }^{*}$ and Zhixing He* ${ }^{*}$
}

\begin{abstract}
Background: Humidity was an unfavorable factor for patients with rheumatoid arthritis (RA). RA disease activity was severe in high humidity conditions. However, there is no evidence to demonstrate the effects of humidity on arthritis in the animal experiments and explore its relevant mechanism.

Methods: Using the DBA/1 mice, this study addressed the effects of a high humidity ( $80 \pm 5 \%$ ) on arthritis in collagen-induced arthritis (CIA) mice. Then, this study used the gas chromatography-mass spectrometer (GC-MS) to explore alterations in serum metabolome caused by the high humidity. Furthermore, xylitol and L-pyroglutamic acid, which were both significantly upregulated by the high humidity, were selected to further study their effects on arthritis in the CIA mice.

Results: The high humidity ( $80 \pm 5 \%$ ) could aggravate arthritis variables including increasing arthritis score and swelling, serum autoantibodies (anti-COII and anti-CCP), and proinflammatory cytokines (IL-6, IL-17A, and G-CSF). In addition, the high humidity could cause significant alterations in serum metabolome in the CIA mice. Xylitol and L-pyroglutamic acid were the representative serum metabolites that were significantly upregulated by the high humidity. Further experiments demonstrated that the supplementation of $0.4 \mathrm{mg} / \mathrm{mL}$ xylitol in drinking water after inducing the CIA model and $2.0 \mathrm{mg} / \mathrm{mL}$ in drinking water before inducing the CIA model could both aggravate arthritis in the CIA mice.
\end{abstract}

Conclusions: These data demonstrated that high humidity was not beneficial for arthritis development and its mechanism might be associated with xylitol and L-pyroglutamic acid.

Keywords: Rheumatoid arthritis, Humidity, Collagen-induced arthritis, Xylitol, L-pyroglutamic acid, DBA/1 mice

\section{Background}

Rheumatoid arthritis (RA) is a chronic systemic autoimmune disease that arises more frequently in females than males, being predominantly observed in the elderly [1]. The global prevalence of RA was 460 per 100,000 population between 1980 and 2018 , with a $95 \%$ prediction interval $(0.06-1.27 \%)$ [2]. RA is characterized by inflammatory

\footnotetext{
*Correspondence: wengcp@yeah.net; hzx2015@zcmu.edu.cn

${ }^{\dagger}$ Mingzhu Wang and Jiao Chen contributed equally to this work. Institute of Basic Research in Clinical Medicine, School of Basic Medical Sciences, Zhejiang Chinese Medical University, Hangzhou 310053, China
}

changes of the synovial tissue of joints, of cartilage and bone, less frequently, of extra-articular sites [3]. The progression of RA can cause joint erosion, functional loss, and the reduction of overall quality of life [4]. Nowadays, the therapeutic armamentarium of RA has expanded with a plethora of conventional synthetic disease-modifying anti-rheumatic drugs in combination with biologic or targeted synthetic [5]. The above treatments for RA are effective in reducing morbidity and mortality but fail to provide a cure.

RA is a multifactorial disease, where various genetic and environmental factors influence the prevalence of the original author(s) and the source, provide a link to the Creative Commons licence, and indicate if changes were made. The images or other third party material in this article are included in the article's Creative Commons licence, unless indicated otherwise in a credit line to the material. If material is not included in the article's Creative Commons licence and your intended use is not permitted by statutory regulation or exceeds the permitted use, you will need to obtain permission directly from the copyright holder. To view a copy of this licence, visit http://creativecommons.org/licenses/by/4.0/. The Creative Commons Public Domain Dedication waiver (http://creativeco mmons.org/publicdomain/zero/1.0/) applies to the data made available in this article, unless otherwise stated in a credit line to the data. 
disease [6]. Although genetic factors have a clear causal relationship to RA, they only account for $40-50 \%$ of seropositive RA and $20-30 \%$ of seronegative RA [7]. Recently, RA is considered arising based on genetic components, but also the environmental factors play an important role in the progression [3]. Environmental factors such as cigarette smoke [8], dust exposure [9], microbes [10, 11], and dietary [12] appear to affect the incidence of RA. In addition, weather conditions are often considered to be strongly associated with RA symptoms in clinical [13, 14]. Humidity is the often-studied weather variable and can influence joint pain and arthritis inflammation in RA patients $[15,16]$. However, there is a lack of study on how humidity affects the occurrence and progression of RA.

In the present study, to clarify the influence of humidity on the onset and development of arthritis, DBA/1 mice (male, 5 weeks old) were kept in a humid environment with $80 \pm 5 \%$ humidity and were injected with bovine type collagen type II to induce arthritis after 3 weeks. Gas chromatography/mass spectrometry (GC-MS) was employed to analyze the influences of the high humidity on serum metabolites of DBA/1 mice. Next, this study revealed the effects of xylitol and L-pyroglutamate, which are both upregulated by the high humidity, on arthritis in collagen-induced arthritis (CIA) mice. Our results may provide novel insights into the pathogenesis of RA, for which the ambient humidity could be considered a promising factor to manage RA.

\section{Materials and methods}

\section{Animals}

Specific pathogen-free (SPF) grade DBA/1 mice (male, 4 weeks old) were purchased from Shanghai SLAC Laboratory Animal Co., Ltd. All the mice were allowed to be acclimated to our animal facility for one week and then randomly divided into different groups in the SPF environment of Zhejiang Chinese Medical University laboratory animal research center. Mice were housed under a $12 \mathrm{~h} / 12 \mathrm{~h}$ light/dark cycle and constant temperature $\left(25 \pm 1{ }^{\circ} \mathrm{C}\right)$ and humidity $(50 \pm 5 \%)$ with food and water available ad libitum. All animal experiments were performed according to the requirements of the Institutional Animal Care and Use Committee of China.

\section{Experimental l: the effect of humidity on arthritis}

The DBA/ 1 mice were grouped into three groups $(N=7)$ : (1) control group (CT): kept in $50 \pm 5 \%$ humidity environment and injected with $0.9 \% \mathrm{NaCl}$ solution on days 21 and 42; (2) CIA group (MT): kept in $50 \pm 5 \%$ humidity environment and injected with $200 \mu \mathrm{g}$ bovine type collagen type II (Chondrex, Redmond, WA, USA) in $200 \mu \mathrm{L}$ complete Freund's adjuvant on day 21 and injected with $200 \mu \mathrm{g}$ bovine type collagen type II in $200 \mu \mathrm{L}$ incomplete
Freund's adjuvant on day 42; (3) humidity CIA group (HT): kept in $80 \pm 5 \%$ humidity environment and the injection method with bovine was the same as the CIA group. The reasons of choosing $80 \%$ humidity were as follows: (1) the $80 \%$ humidity was regarded as the boundary of high humidity in the previous literatures [17-20]; (2) the $80 \%$ humidity could not affect the intake of diet and water in DBA/1 mice. The time course, grouping information and humidity fluctuations were shown in Fig. 1. The entire experimental period was 8 weeks.

The blood was obtained from the eye socket vein on days 21,42 , and 56 and then centrifuged at $3000 \mathrm{rpm}$ for 15 min at $4{ }^{\circ} \mathrm{C}$ for serum. The arthritis scores and ankle joint swelling were measured every 3 days after day 42 . The DBA/1 mice with 15 weeks old were euthanized to obtain the hind limbs of mice including the ankle.

\section{Experimental II: the effect of xylitol or L-pyroglutamate on arthritis}

After clarifying the effect of the high humidity ( $80 \pm 5 \%)$ on the CIA model, this study selected xylitol and L-pyroglutamate, which were up-regulated by the high humidity according to serum metabolome analysis, for further study their effects on the CIA model. The DBA/1 mice were grouped into ten groups $(N=7)$ and the detailed group information was shown in Fig. 2. Both xylitol and L-pyroglutamate were administrated in drinking water. The entire experimental period was 8 weeks.

The blood was obtained from the eye socket vein on day 56 and then centrifuged at $3000 \mathrm{rpm}$ for $15 \mathrm{~min}$ at $4{ }^{\circ} \mathrm{C}$ for serum. The arthritis score and ankle joint swelling were measured every 3 days after day 42 . The DBA/ 1 mice with 16 weeks old were euthanized to obtained the hind limbs of mice including the ankle.

\section{Assessment of arthritis symptoms}

The arthritis symptoms, including arthritis scores and ankle joint swelling, were measured every 3 days after the second collagen injection. The arthritis severity in each mouse paw was scored every alternate day in a blinded manner according to the marginally modified method of Moore et al. and Khachigian [21, 22]. The arthritis scores were assessed as follows: 0 , no swelling and redness in the foot joints; 1 , mild swelling and redness of the little toe joint; 2, moderate swelling and redness of the toe joint and plantar joint; 3, paw swelling below the ankle joint; 4, all paw swelling including ankle joint is 4 points; The total score of each limb was added to the total arthritis score of the mouse. The maximum possible score for each mouse was calculated as 16 in this study design. The ankle joint swelling was quantified by measuring paw volume using a YSC-7C paw volume meter (Shandong Academy of Medical Sciences, Jinan, China). 


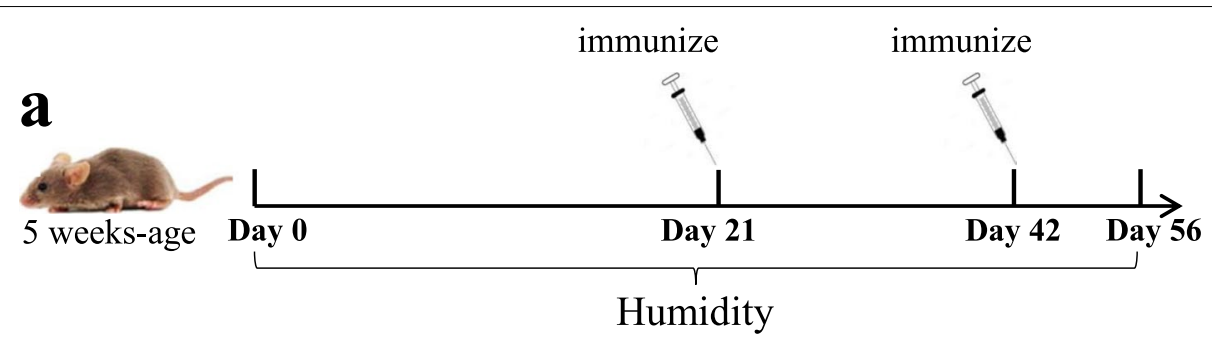

b

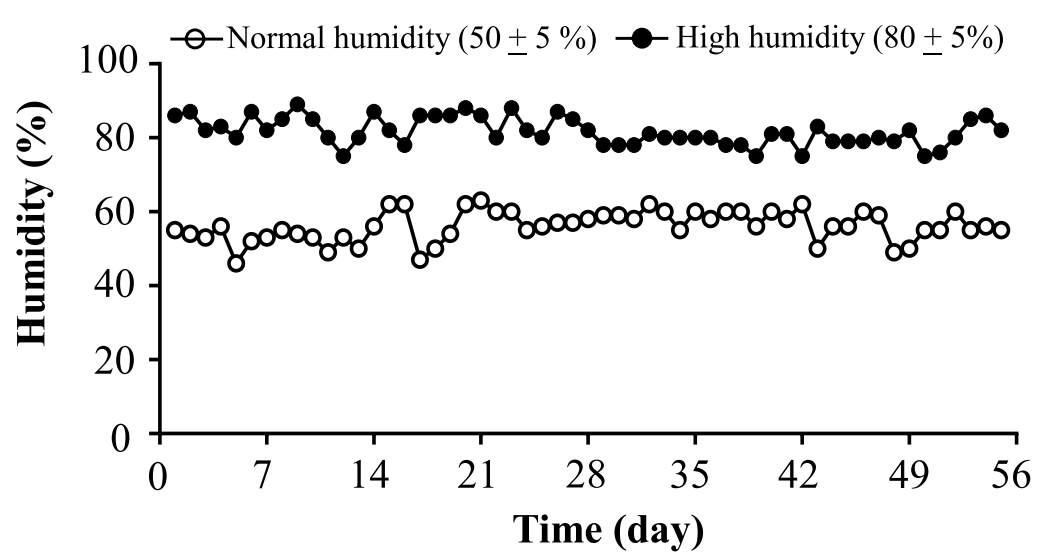

Fig. 1 a The diagram of the experimental treatments is shown. $\mathbf{b}$ Variation of humidity in the cage per day

The ankle tissue was also harvested from exsanguinated mice, flushed with $1 \times$ PBS, dissected longitudinally, and fixed in $4.0 \%$ formaldehyde overnight, and decalcified in EDTA decalcification solution. The tissues were then embedded in paraffin. Sections of $5 \mu \mathrm{m}$ were cut from paraffin-embedded tissues and stained with hematoxylin and eosin $(H \& E)$ to evaluate the damage of ankle tissue.

\section{Measurements of serum autoantibodies and pro-inflammatory cytokines}

Serum type II collagen antibody (Col II) and anti-cyclic citrullinated peptide antibody (anti-CCP) were evaluated using CUSABIO ELISA Kits (Wuhan, China) that was based on the double antigen sandwich ELISA method. In Experiment I, serum levels of pro-inflammatory cytokines were measured by a commercial multiplex mouse cytokine magnetic bead-based immunoassay (BioPlex Pro Mouse Cytokine 23-plex Assay, Bio-Rad Laboratories) according to the manufacturer's instructions. The cytokine screen included IL- $1 \alpha$, IL-1 $\beta$, IL-2, IL-3, IL-4, IL-5, IL-6, IL-9, IL-10, IL-12p40, IL-12p70, IL-13, IL-17A, eotaxin, G-CSF, GM-CSF, IFN- $\gamma, \mathrm{KC}, \mathrm{MCP}-1$, MIP-1 $\alpha$, MIP-1 $\beta$, RANTES, and TNF- $\alpha$. The mean fluorescence intensity from all the bead combinations tested was analyzed using the Bio-Plex system equipped with Bio-Plex Manager Software v6.0 (Bio-Rad Laboratories). In Experiment II, serum levels of IL-6, IL-17A, G-CSF, and eotaxin were evaluated using the Multisciences ELISA Kits (Hangzhou, China) according to the manufacturer's instructions.

\section{GC-MS analysis conditions and data processing}

Serum samples were prepared according to the previous method [23]. After thawing on ice, $50 \mu \mathrm{L}$ of serum sample was placed in a $1.5 \mathrm{~mL}$ Eppendorf vial and kept on ice, subsequently, $200 \mu \mathrm{L}$ cold methanol with $5 \mathrm{mg} /$ $\mathrm{mL}$ hexadecanoic acid (Sigma, internal standard) was added. After vortexing for $30 \mathrm{~s}$, the sample was kept standing for $10 \mathrm{~min}$, and then centrifuged for $10 \mathrm{~min}$ to precipitate the protein $\left(12,000 \mathrm{rpm}, 4{ }^{\circ} \mathrm{C}\right)$. Then, $185 \mu \mathrm{L}$ supernatant was transferred to a new $1.5 \mathrm{~mL}$ Eppendorf vial and dried under a vacuum condition produced by a Labconco CentriVap (Labconco, Kansas, MO, USA). Fifty microliters of methoxyamine pyridine (Aldrich) solution $(20 \mathrm{mg} / \mathrm{mL})$ were added to the dried residue to re-dissolve, and the resuspended solution was vortexed for 30 s, ultrasonic for $10 \mathrm{~min}$ and oximated for $90 \mathrm{~min}$ in a 37 ${ }^{\circ} \mathrm{C}$ water bath. Afterward, $50 \mu \mathrm{L}$ of MSTFA (Sigma) was added for trimethylsilylation for $60 \mathrm{~min}$. After a series of the above-mentioned processing, the supernatant was prepared for analysis. A quality control (QC) strategy was applied to monitor the variability within an analytical batch and ensure the data quality. QC sample was prepared by equally mixing the sera from all groups, and 


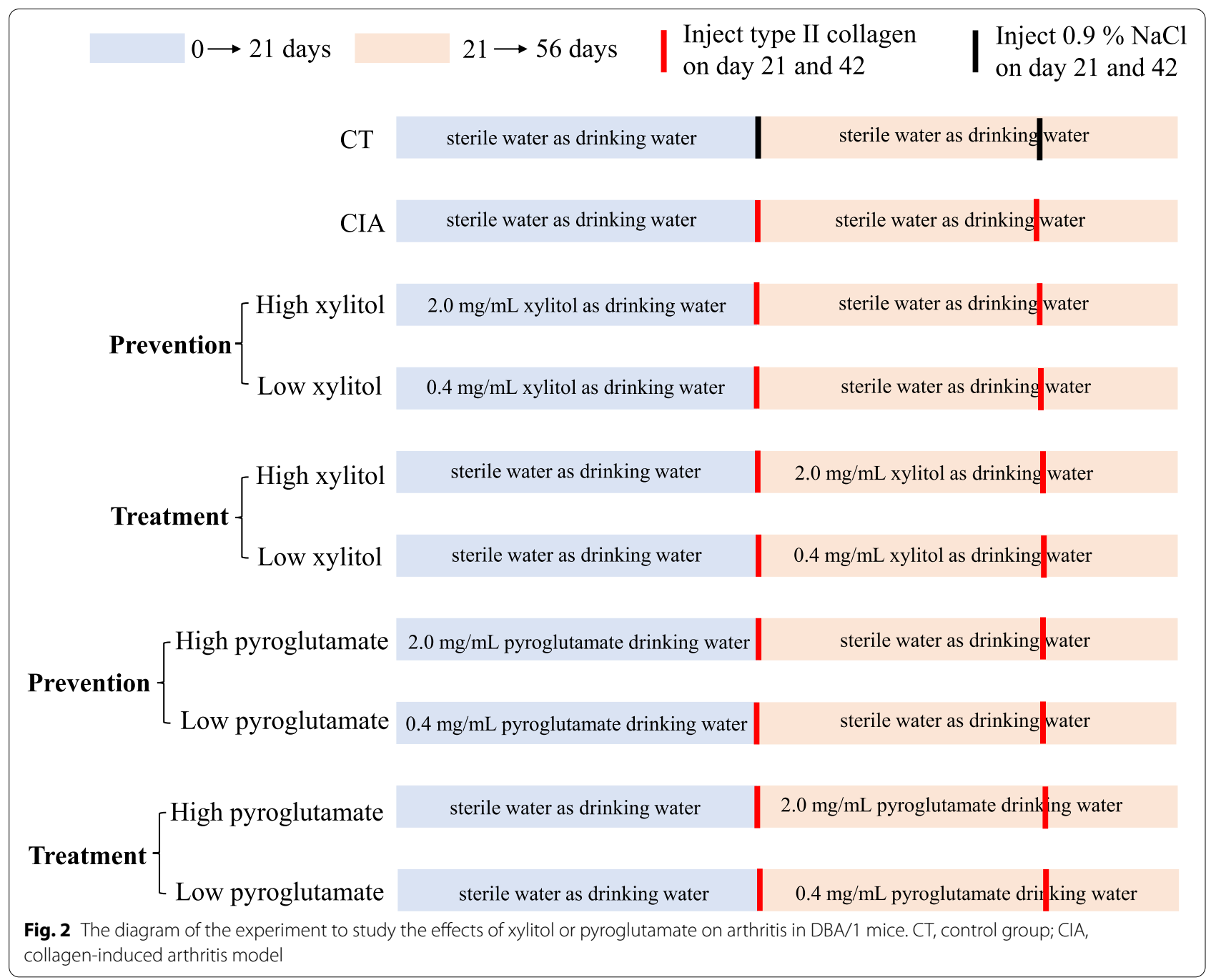

QCs were processed together with samples by using the same method.

Metabolite analysis was carried out by SHIMADZU GCMS-QP2010 GC/MS (SHIMADZU Corporation, Japan). Separation was performed by loading a $30-\mathrm{m} \times$ $0.25-\mathrm{mm} \times 0.25-\mu \mathrm{m}$ HP-5MS fused silica capillary column (Agilent J\&W Scientific). The inlet and ion source temperatures were $300{ }^{\circ} \mathrm{C}$ amd $230{ }^{\circ} \mathrm{C}$, respectively. The flow rate of the carrier gas, high-purity helium (>99.999\%), was $1.2 \mathrm{~mL} / \mathrm{min}$. The sample injection volume was $1 \mu \mathrm{L}$ with a split ratio of $10: 1$. The $\mathrm{GC}$ oven temperature program consisted of $70{ }^{\circ} \mathrm{C}$ for $3 \mathrm{~min}$, after which the temperature ramped to $300{ }^{\circ} \mathrm{C}$ at $5{ }^{\circ} \mathrm{C} / \mathrm{min}$, and held steady for $5 \mathrm{~min}$. The mass spectrum scan range was set at $50-550 \mathrm{~m} / \mathrm{z}$, and the acquisition frequency was 2 $\mathrm{Hz}$ after a solvent delay of $4.8 \mathrm{~min}$. A dispersion analysis was performed for different groups of samples, with one quality control (QC) sample inserted for every five samples to measure system drift.
Raw GC-MS mass spectra were converted to CDF format files and subsequently was performed feature extraction and preprocessed with XCMS in R software as previously described [24]. The data were normalized and edited into a two-dimensional data matrix by Excel 2010 software, including retention time (RT), mass-to-charge ratio (MZ), observations (samples), and peak intensity. Identification of metabolites was conducted using the Automatic Mass Spectral Deconvolution and Identification System (AMIDS), which was searched against commercially available databases such as the National Institute of Standards and Technology (NIST) and Wiley libraries. Metabolites were identified by comparison of mass spectra and retention indices to the spectral library using matching values greater than 80 . The signal integration area of each metabolite was normalized to the internal standard (hexadecanoic acid) for each sample. Typical GC-MS spectra of serum metabolites obtained from DBA/1 mice were shown in Fig. S1. As shown in 
Table S1, 58 targeted metabolites were confirmed on the basis of their retention and MS fragmentation behavior.

For multivariate statistical analysis, the XCMS output was further processed using Microsoft Excel (Microsoft, USA). The normalized data were transformed using SIMCA-P 11.0 software (Umetrics AB, Umea, Sweden) for partial least Squares-discriminant analysis (PLS-DA). PLS-DA was applied to the data after mean-centering and unit variance scaling (UV scaling). These analyses employed a default sevenfold internal cross-validation from which the $R^{2} X$ and $Q 2$ (goodness of prediction) values, representing the total explained variance and the model predictability, respectively, were extracted. The variable importance in projection (VIP) values of all the metabolites from the PLS-DA model were taken as criteria to find the variable importance of differential metabolites. Those variables with a VIP $>1.0$ and a $p$ value $<0.05$ were considered relevant for group discrimination.

\section{Statistical analysis}

The statistical significance between the two groups was evaluated by a univariate Student's $t$ test (SPSS 22, International Business Machines Corp., Armonk, USA)). Following statistical analyses with multiple comparisons, the $p$ value was adjusted using the Benjamini-Hochberg method to control the false discovery rate (FDR). An adjusted $p$ value of 0.05 was used as a statistically significant cutoff.

\section{Results}

\section{High humidity aggravated arthritis symptoms in the $\mathrm{CIA}$ model mice}

To directly assess the effects of humidity on inflammatory arthritis in vivo, a CIA model was established in DBA/1 mice living in normal (50 $\pm 5 \%$ ) humidity or high $(80 \pm$ $5 \%)$ humidity. Compared to the normal mice, CIA mice under both two humidities showed joint swelling, inflammation and destruction, and increased autoantibodies after the second immunization (Fig. 3, Table S2). The following analysis was to compare the difference in arthritis outcomes between the normal and high humidity. The results showed that CIA mice under the high humidity had significantly higher arthritis scores at days 50 and 55, more severe ankle swelling at days 42 and 46 than that of CIA mice under normal humidity (Fig. 3a, b, Table S2). In addition, both autoantibodies (anti-COII IgG and antiCCP) analysis indicated that the high humidity could significantly increase autoantibodies of the CIA mice on day 56 , but had no effect on day 42 in the comparison with the normal humidity (Fig. 3c, d, Table S2). The H\&E histology of the CIA mice also showed that the high humidity could cause more severe synovial inflammation than normal humidity (Fig. 3e, f). Therefore, the high humidity could aggravate arthritis in the development of CIA of DBA/1mice.

\section{Humidity induced more severe inflammation in the CIA model mice}

We then investigated 23 serum proinflammatory cytokines to reflect inflammation of the CIA mice (Fig. 4, Fig. S2, Table S3). Before the CIA model was established, the exposure to the high humidity for 21 days did not cause the upregulation of inflammatory cytokines in DBA mice (Fig. 4, Fig. S2, Table S3). After the CIA model was established, three comparisons were conducted, including $\mathrm{CT}$ vs. MT, CT vs. HT, and MT vs. HT. The proinflammatory cytokines that exhibited no statistical difference in all three comparisons included IL-1a, IL-1b, IL-3, MCP-1, and TNF-a (Fig. S2, Table S3).

The results of comparisons on day 42 were described as follows. Compared to the CT group, both MT and HT had significantly increased levels of IL-12 p40, IFN-g, RANTES, IL-6, IL-17A, G-CSF, and eotaxin, decreased levels of IL-2 and IL-5 (Fig. 4, Fig. S2, Table S3). Besides, compared to the CT group, IL-10 and IL-12 p70 significantly decreased, KC significantly increased in the HT group but not in the MT group (Fig. S2, Table S3). In the comparison between MT and HT, IL-10 significantly decreased and IL-13, IL-6, IL-17A, and G-CSF significantly increased in the HT group (Fig. 4, Fig. S2, Table S3).

Next, this study also showed the results of pro-inflammatory cytokines on day 56. Compared to the CT group, only MIP-1b, IL-6, G-CSF, and eotaxin were significantly increased in the both MT and HT groups (Fig. 4, Fig. S2, Table S3). In addition, the HT group had lower levels of IL-4 and MIP-1a, and higher levels of IL-9, IL-6, IL-17A, and G-CSF than the MT group (Fig. 4, Fig. S2, Table S3).

Taken together, the high humidity could aggravate collagen-induced upregulation of IL-6, IL-17A, and G-CSF in the development of the CIA model.

\section{Effects of humidity on serum metabolome of the CIA model mice}

To uncover serum metabolite differences by humidity, a comparative PLS-DA model based on the targeted metabolites was conducted between $\mathrm{CT}$ and $\mathrm{HT}$ on day 21, between MT and HT on day 42, and between MT and HT on day 56. The values for $R^{2} X$ and $Q 2$ (Fig. 5a, c, e) and the results of permutation tests (Fig. S3) indicated the goodness of fit and predictability of the models in revealing the humidity-induced alterations in serum metabolites in DBA/1 mice. High humidity caused clear separations between two groups in all three PLS-DA score plots (Fig. 5a, c, e). Metabolites with a VIP value greater than 1.0 were displayed with bigger and color 

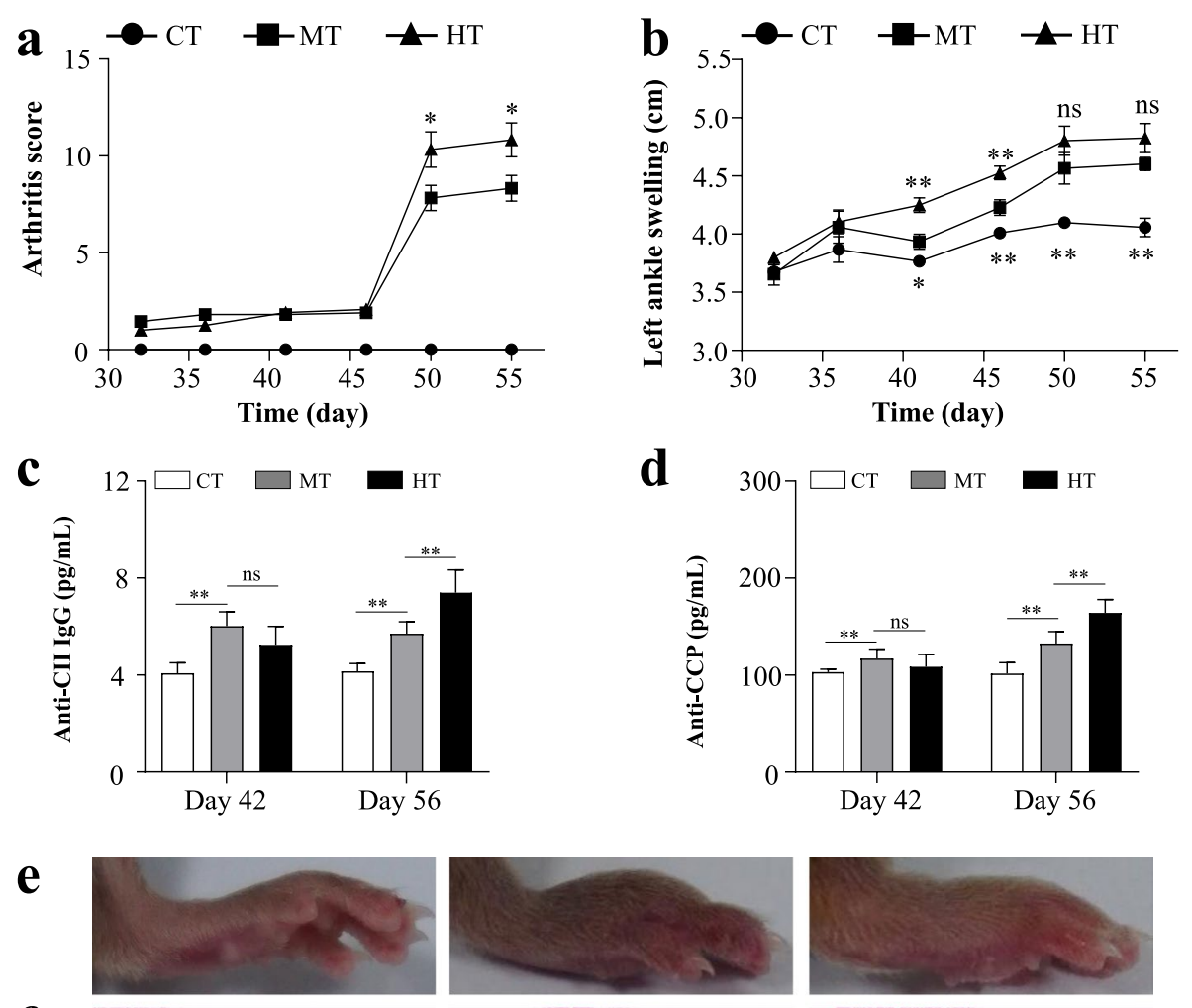

f
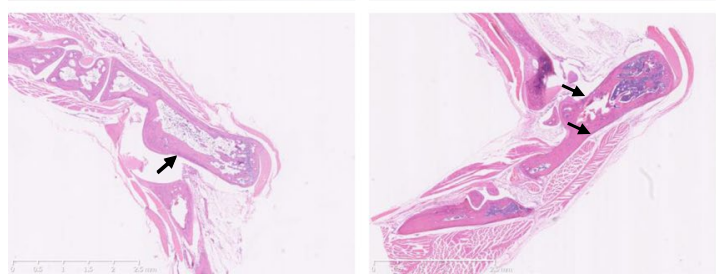

CT

MT

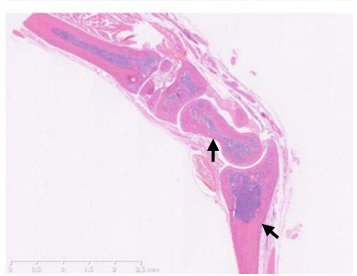

HT

Fig. 3 High humidity aggravates the severity of collagen-induced arthritis (CIA). a Arthritis scores were assessed every five days starting after day 32. b Left ankle swelling was assessed every five days starting after day 32. c Serum anti-CII lgG was measured by ELISA on days 42 and 56 . d Serum anti-CCP was measured by ELISA on days 42 and 56. e Representative images of ankle joint. $\mathbf{f}$ Representative images of H\&E stained histological of ankle joint. Values are the mean $\pm \mathrm{SEM} .{ }^{\prime * *}=P<0.05 ;{ }^{\prime * * "}=P<0.01, " \mathrm{~ns} "=P \geq 0.05$. CT, control group; MT, inducing collagen-induced arthritis group under $50 \%$ humidity; $H$ T, inducing collagen-induced arthritis group under $80 \%$ humidity

triangles in three corresponding loading plots (Fig. 5b, d, f) and were considered the primary contributors for the classification of the two groups.

In combination with $p$ ( $t$ test) and VIP values, four serum metabolites significantly altered between CT and HT group before inducing CIA (day 21). The high humidity could cause significant increases of xylitol, dodecanoic acid, and L-pyroglutamic acid, a decrease of sucrose in DBA/1 mice before inducing CIA (day 21) (Table 1). Before the second injection of collagen (day 42), the high humidity caused significant increases of xylitol, L-pyroglutamic acid, and L-threonine, a decrease of dodecanoic acid, malic acid, and D-gluconic acid in $\mathrm{DBA} / 1$ mice (Table 1). In addition, there were 15 differential metabolites between MT and HT groups at day 56. Compared with the MT group, the HT group had a significantly higher concentration of 12 serum metabolites, such as xylitol, pyroglutamic acid, L-aspartic acid, and glucose oxime, lower concentration of 3 serum metabolites, including L-valine, L-alanine, and pyridine (Table 1).

According to the above results, xylitol and L-pyroglutamic acid were significantly elevated by the high humidity at all time points.

\section{Effects of xylitol on arthritis in the CIA model mice}

Xylitol was significantly upregulated in CIA model mice due to the high humidity. Next, this study explored the 

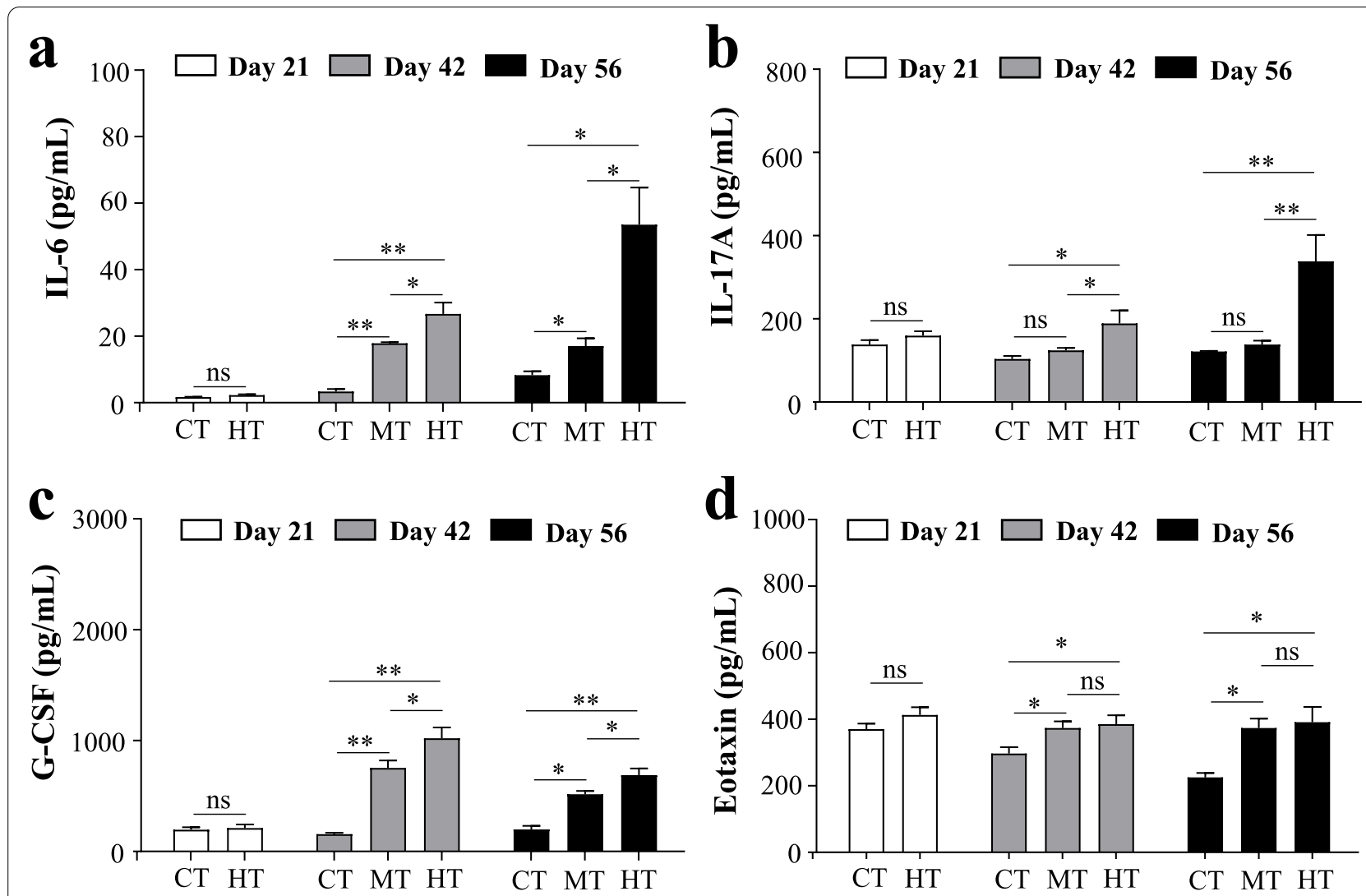

Fig. 4 Serum proinflammatory cytokines levels of mice in the three groups. a IL-6; $\mathbf{b}$ IL-17A; $\mathbf{c}$ G-CSF; $\mathbf{d}$ eotaxin. Values are the mean + SEM. ${ }^{\prime * *}=P<0.05 ;{ }^{\prime * * *}=P<0.01, " n s^{\prime \prime}=P>0.05$. CT, control group; MT, inducing collagen-induced arthritis group under $50 \%$ humidity; $\mathrm{HT}$, inducing collagen-induced arthritis group under $80 \%$ humidity

effects of xylitol on arthritis in CIA model mice to further reveal the mechanisms of humidity environment aggravating arthritis. As shown in Fig. 6, only a low dose of xylitol given after inducing CIA could significantly upregulate the levels of anti-COII IgG, IL-6, IL-17A, and arthritis score. Interestingly, G-CSF and eotaxin were significantly decreased by high-dose of xylitol given before inducing the CIA model (Fig. 6, Table S4). In sum, a low dose of xylitol given after inducing CIA could aggravate arthritis in CIA model mice.

\section{Effects of L-pyroglutamate on arthritis in the ClA model mice}

L-pyroglutamate was another significantly increased serum metabolite, which was caused by the high humidity in CIA model mice. As shown in Fig. 7 and Table S5, a high dose of L-pyroglutamate given before inducing CIA could significantly upregulate anti-COII IgG, IL-6, eotaxin, and arthritis score. Besides, G-CSF was significantly down-regulated in all four pyroglutamate groups (Fig. 7, Table S5). Eotaxin was significantly downregulated by a low dose of pyroglutamate given before inducing CIA and a high-dose of pyroglutamate given after inducing CIA (Fig. 7, Table S5). Taken together, a high dose of pyroglutamate given before inducing CIA could aggravate arthritis in CIA model mice.

\section{Discussion}

Environmental factors have been associated with an increased risk for RA [6, 25]. Humidity was an unfavorable factor for RA's occurrence and progression, but the above conclusion was only demonstrated in clinical RA patients. So far, there was no experimental study to prove the influence of humidity on RA and its relevant mechanism. This study is the first to prove that a high humidity $(80 \pm 5 \%)$ could significantly aggravate arthritis in the CIA model mice and explore the related mechanism from the perspective of serum metabolites.

Humidity was included as an environmental factor in a wide range of human health topics [26]. Conceptually, the humidity was linked with anomalous mortality and morbidity levels through its role in affecting heat stress and hydration state [27]. Therefore, humidity could be considered as a direct or indirect trigger for several 

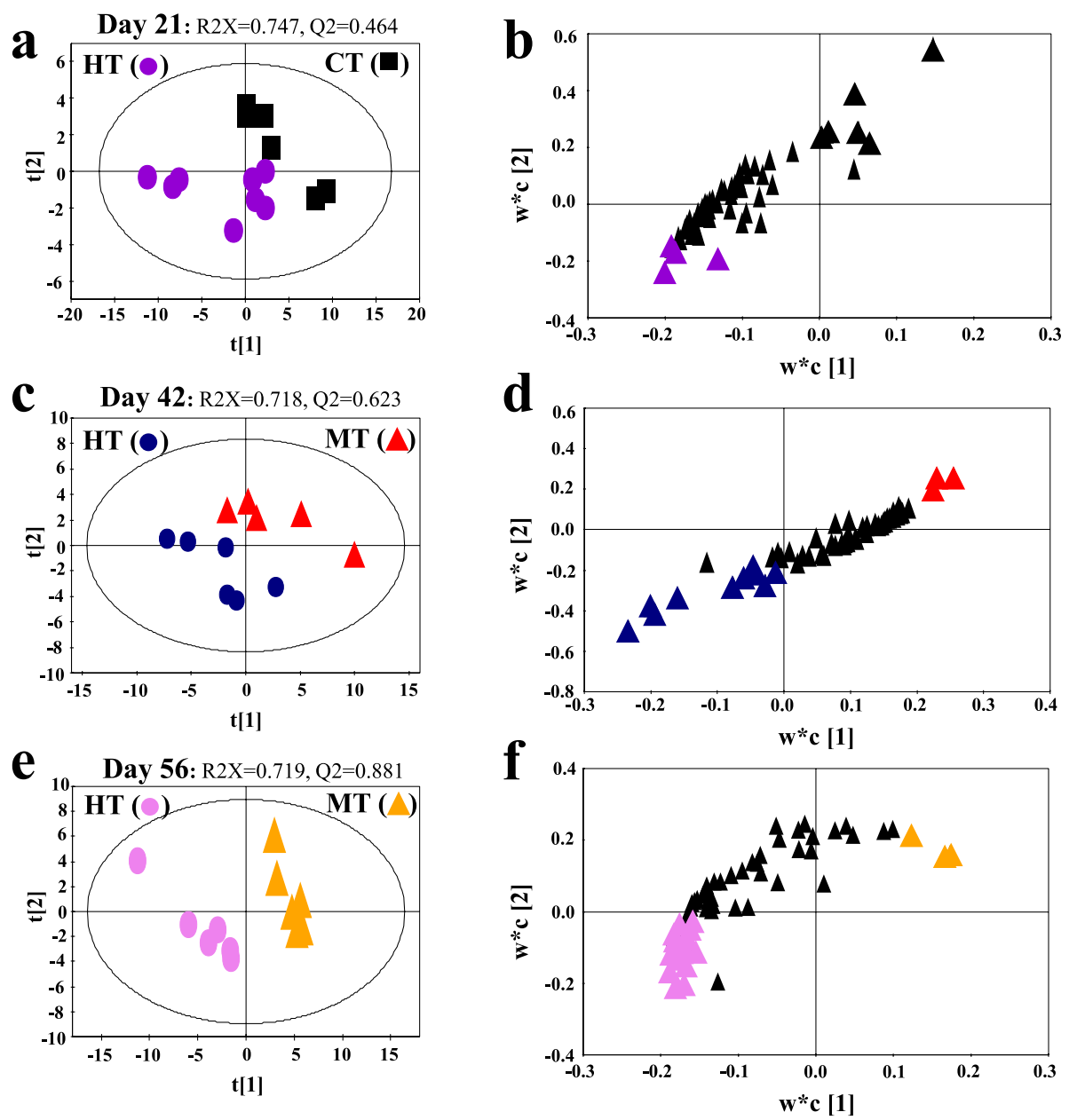

Fig. 5 PLS-DA score $(\mathbf{a}, \mathbf{c}, \mathbf{e})$ and loading plots $(\mathbf{b}, \mathbf{d}, \mathbf{f})$ based on the serum metabolic profiles of DBA/1 mice. $\mathbf{a}, \mathbf{b}$ Comparison of the results between the humidity group ( $\mathrm{HT}$, purple dot) and control group (CT, black square) at day 12. The purple triangle represents that the metabolite was higher in the HT group than $\mathrm{CT}$; the black triangle represents that the metabolite was higher in the $\mathrm{CT}$ group than HT. $\mathbf{c}$, $\mathbf{d}$ Comparison of the results between the high humidity CIA group (HT, blue dot) and normal humidity CIA model group (MT, red triangle) at day 42 . The blue triangle represents that the metabolite was higher in the HT group than MT; red triangle represents that the metabolite was higher in the MT group than HT. e, $\mathbf{f}$ Comparison of the results between the high humidity CIA group (HT, pink dot) and normal humidity CIA model group (MT, yellow triangle) at day 56. The pink triangle represents that the metabolite was higher in the HT group than MT; the yellow triangle represents that the metabolite was higher in the MT group than HT

diseases, such as cardiovascular [28], pulmonary [29], and zoonotic diseases [30]. The classic opinion, "Cold and wet is bad, warm and dry is good for RA patients" seems to be true as far as humidity is concerned [31]. The humidity could influence RA variables, like pain [14], stiffness [32], and ESR [33] in RA patients. In the CIA model mice, this study successfully demonstrated that the high humidity could aggravate RA variables including increasing arthritis scores and swelling, serum autoantibodies (anti-COII and anti-CCP), and proinflammatory cytokines (IL-6, IL-17A, and G-CSF). As far as we know, this is the first study focusing on the influence of humidity on RA symptoms in animal experiments.
In addition, this study explored the mechanism of high humidity aggravating arthritis from the perspective of serum metabolites. Since metabolites are typically the end-products of the genome, transcriptome, and proteome, alterations in these are indicative of the overall physiological state of the investigated biological system [34]. Serum metabolites that associate with the high humidity might shed light on the underlying pathophysiology of humidity causing arthritis in DBA/1 mice. The longer the high humidity affected, the more altered metabolites the high humidity-induced in DBA/1 mice. This study also showed xylitol and L-pyroglutamic acid that were reproducibly associated with the high humidity 
Table 1 Significantly altered serum metabolites related to humidity in DBA/1 mice

\begin{tabular}{|c|c|c|c|c|c|c|c|c|c|}
\hline & \multicolumn{3}{|c|}{ Day 21 - HT vs. CT } & \multicolumn{3}{|c|}{ Day 42 - HT vs. MT } & \multicolumn{3}{|c|}{ Day 56 - HT vs. MT } \\
\hline & VIP & $P$ value & Trend & VIP & $P$ value & Trend & VIP & $P$ value & Trend \\
\hline Xylitol & 1.40 & 0.004 & $\uparrow$ & 1.87 & 0.043 & $\uparrow$ & 1.21 & 0.000 & $\uparrow$ \\
\hline L-aspartic acid & 1.04 & $>0.05$ & - & $<1.0$ & $>0.05$ & - & 1.36 & 0.000 & $\uparrow$ \\
\hline Dodecanoic acid & 1.09 & 0.007 & $\uparrow$ & 1.10 & 0.019 & $\downarrow$ & $<1.0$ & 0.007 & - \\
\hline Pyroglutamic acid & 1.10 & 0.014 & $\uparrow$ & 1.70 & 0.038 & $\uparrow$ & 1.11 & 0.002 & $\uparrow$ \\
\hline Sucrose & 2.86 & 0.034 & $\downarrow$ & $<1.0$ & $>0.05$ & - & $<1.0$ & $>0.05$ & - \\
\hline L-tryptophan & 2.07 & $>0.05$ & - & $<1.0$ & $>0.05$ & - & $<1.0$ & 0.008 & - \\
\hline Ethanolamine & 1.33 & $>0.05$ & - & $<1.0$ & $>0.05$ & - & $<1.0$ & $>0.05$ & - \\
\hline Triethylene glycol & 1.11 & $>0.05$ & - & $<1.0$ & $>0.05$ & - & $<1.0$ & $>0.05$ & - \\
\hline L-threonine & 1.37 & $>0.05$ & - & 2.27 & 0.015 & $\uparrow$ & $<1.0$ & $>0.05$ & - \\
\hline Galactonic acid & 1.31 & $>0.05$ & - & $<1.0$ & $>0.05$ & - & $<1.0$ & $>0.05$ & - \\
\hline L-valine & $<1.0$ & $>0.05$ & - & 1.52 & $>0.05$ & - & 1.20 & 0.001 & $\downarrow$ \\
\hline Methyl galactoside & $<1.0$ & 0.011 & - & 1.39 & $>0.05$ & - & 1.36 & 0.000 & $\uparrow$ \\
\hline L-isoleucine & $<1.0$ & $>0.05$ & - & 1.20 & $>0.05$ & - & $<1.0$ & $>0.05$ & - \\
\hline Glucose oxime & $<1.0$ & 0.013 & - & 1.49 & $>0.05$ & - & 1.17 & 0.001 & $\uparrow$ \\
\hline L-alanine & $<1.0$ & $>0.05$ & - & 1.07 & $>0.05$ & - & 1.01 & 0.023 & $\downarrow$ \\
\hline Malic acid & $<1.0$ & $>0.05$ & - & 1.24 & 0.017 & $\downarrow$ & $<1.0$ & $>0.05$ & - \\
\hline D-gluconic acid & $<1.0$ & 0.023 & - & 1.31 & 0.007 & $\downarrow$ & 1.16 & 0.000 & $\uparrow$ \\
\hline D-mannose & $<1.0$ & $>0.05$ & - & 1.15 & $>0.05$ & - & 1.27 & 0.000 & $\uparrow$ \\
\hline Methyltrifluoroacetamide & $<1.0$ & 0.039 & - & $<1.0$ & $>0.05$ & - & 1.28 & 0.000 & $\uparrow$ \\
\hline Octadecanoic acid & $<1.0$ & $>0.05$ & - & $<1.0$ & $>0.05$ & - & 1.31 & 0.000 & $\uparrow$ \\
\hline Campesterol & $<1.0$ & 0.033 & - & $<1.0$ & $>0.05$ & - & 1.25 & 0.000 & $\uparrow$ \\
\hline 1-monopalmitin & $<1.0$ & 0.022 & - & $<1.0$ & $>0.05$ & - & 1.26 & 0.000 & $\uparrow$ \\
\hline Glycerol monostearate & $<1.0$ & $>0.05$ & - & $<1.0$ & $>0.05$ & - & 1.23 & 0.000 & $\uparrow$ \\
\hline Pyridine & $<1.0$ & 0.034 & - & $<1.0$ & $>0.05$ & - & 1.25 & 0.000 & $\downarrow$ \\
\hline
\end{tabular}

Note: " $\uparrow$ " represents the significant increase caused by humidity; “ $\downarrow$ " represents the significant caused by humidity, and "—" represents no significant alteration caused by humidity. CT, control group; $M T$, inducing collagen-induced arthritis group under $50 \%$ humidity; $H T$, inducing collagen-induced arthritis group under $80 \%$ humidity

before and after inducing the CIA model. Therefore, xylitol and L-pyroglutamic acid should be closely associated with the aggravation of arthritis by the high humidity in CIA mice.

To further reveal the influences of serum metabolites significantly altered by high humidity on arthritis, this study selected xylitol and L-pyroglutamic acid for further study based on VIP and $p$ values. The results indicated that the effect of xylitol or L-pyroglutamic acid on arthritis was closely associated with the administration time and dose in the CIA model mice. After inducing the CIA model, supplementation of $0.4 \mathrm{mg} / \mathrm{mL}$ xylitol in drinking water could aggravate arthritis in the CIA model mice. However, the previous literature has reported that a $10 \%$ dietary xylitol supplementation could protect against the imbalance in bone metabolism during the early phase of collagen type II-induced arthritis in rats [35]. The inconsistent effects of xylitol might be due to the concentration of xylitol and the period of collagen-induced arthritis. Unlike xylitol, L-pyroglutamic acid could aggravate arthritis when it was supplemented with $2.0 \mathrm{mg} / \mathrm{mL}$ in drinking water before inducing the CIA model. This study explored the influences of L-pyroglutamic acid on arthritis for the first time. Although there was no study revealing the correlation between RA and L-pyroglutamic acid, increased L-pyroglutamic acid could be considered as a potential diagnostic biomarker for systemic lupus erythematosus or nonalcoholic steatohepatitis in clinical [36, 37]. In addition, L-pyroglutamic acid could be an indicator of toxicity [38]. Overall, both xylitol and L-pyroglutamic acid increased by high humidity could aggravate arthritis, but these effects depended on the administration time and dose of them.

\section{Conclusions}

In conclusion, our data revealed that a high humidity (80 $\pm 5 \%)$ could aggravate arthritis and its mechanism might be associated with xylitol and L-pyroglutamic acid. However, the limitations of this study were as follows: (1) this study did not validate the results through human samples and further the mechanism of the influence mechanism of xylitol and L-pyroglutamic acid; (2) this study 

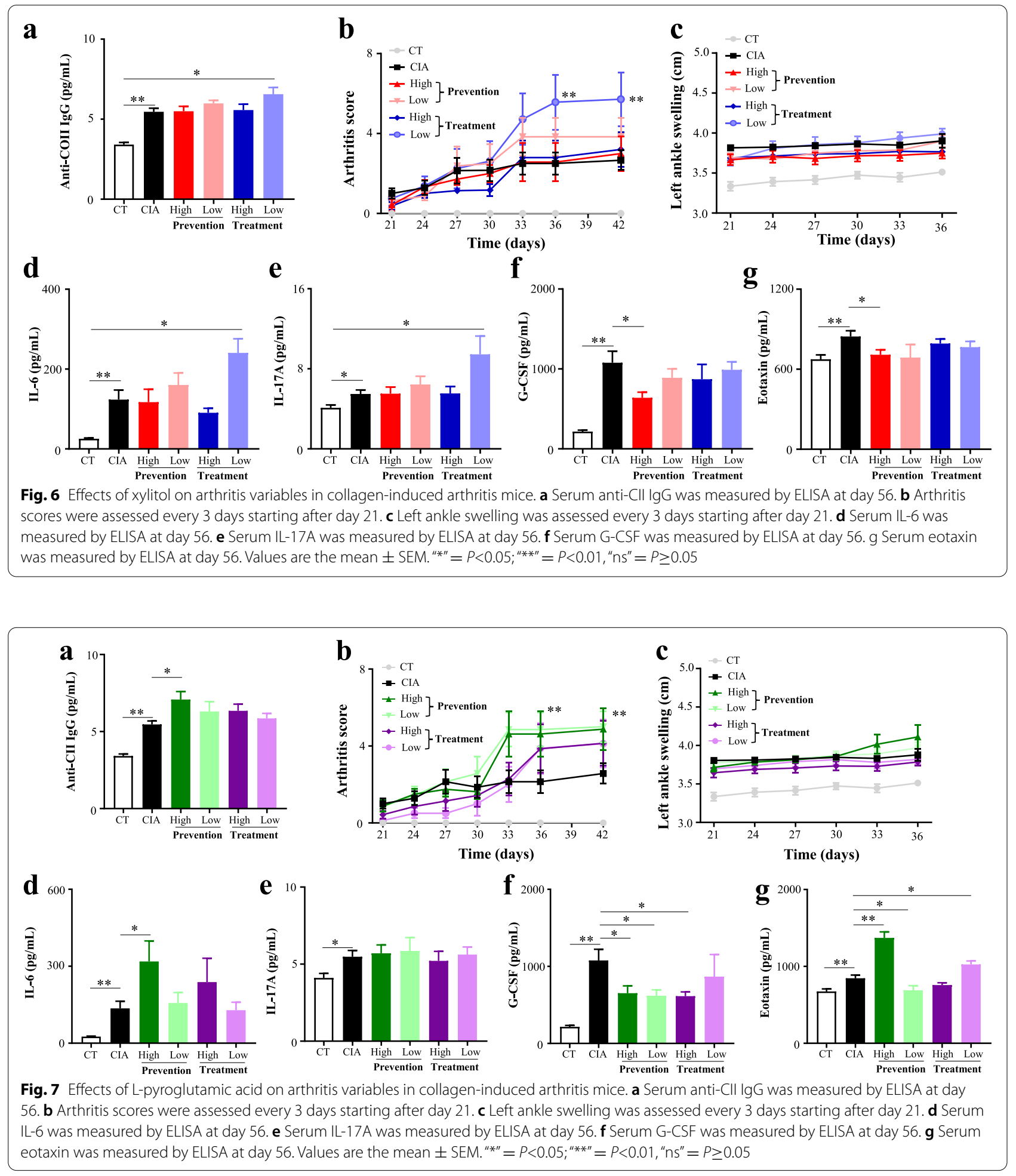

did not calculate the inter-rater reliability or intra-rater of arthritis scores between authors; (3) this study did not record the cage temperature vales throughout the experiment. Even so, this study could facilitate the deep understanding of the relationship between humidity environment on RA. 


\section{Abbreviations}

RA: Rheumatoid arthritis; CIA: Collagen-induced arthritis; GC-MS: Gas chromatography-mass spectrometer; anti-COII: Anti-collagen type II antibody; anti-CCP: Anti-cyclic citrullinated peptide antibody; QC: Quality control; VIP: Variable importance in projection; FDR: False discovery rate; CT: Control group; MT: Collagen-induced arthritis model group under normal humidity; HT: Collagen-induced arthritis model group under high humidity; PCA: Principal component analysis; PLS-DA: Partial least Squares-discriminant analysis; SPF: Specific pathogen-free.

\section{Supplementary Information}

The online version contains supplementary material available at https://doi. org/10.1186/s13075-021-02681-x.

\section{Additional file 1: Figure S1 \\ Additional file 2: Figure S2. \\ Additional file 3: Figure S3. \\ Additional file 4: Table S1 List of serum metabolites found in GC-MS analysis.}

Additional file 5: Table S2 The numeric values of CIA measurements (mean \pm SE) in Fig. 3.

Additional file 6: Table S3 The numeric values of CIA measurements (mean \pm SE) in Fig. 4 .

Additional file 7: Table S4 The numeric values of CIA measurements (mean \pm SE) in Fig. 6 .

Additional file 8: Table S5 The numeric values of CIA measurements (mean \pm SE) in Fig. 7 .

\section{Acknowledgements}

Not applicable

\section{Authors' contributions}

$\mathrm{ZH}$ and $\mathrm{CW}$ contributed toward conceiving the research. $\mathrm{MW}, \mathrm{JC}$, and $\mathrm{XL}$ conducted animal experiments. $\mathrm{LH}, \mathrm{HL}$, and $\mathrm{ZH}$ assisted in conducting the experiments. $\mathrm{ZH}, \mathrm{MW}$, and $\mathrm{HL}$ analyzed the data and drafted the manuscript. All authors contributed to the article and approved the submitted version.

\section{Funding}

This work was supported by the National Key Research and Development Program of China (No. 2018YFC1705501), the National Natural Science Foundation of China (No. 82074217), the Natural Science Foundation of Zhejiang Province (No. LY21H270006).

\section{Availability of data and materials}

Further information and requests for resources should be directed to the corresponding author, Z.H. (hzx2015@zcmu.edu.cn)

\section{Declarations}

\section{Ethics approval and consent to participate}

All animal handling and experimental procedures were performed following local ethical committees and the National Institutes of Health Guide for the Care and Use of Laboratory Animals. All efforts were made to minimize animal suffering and to reduce the number of animals used. All procedures performed in this study involving animals were approved by the Ethics Committee of Zhejiang Chinese Medical University.

\section{Consent for publication}

Not applicable

\section{Competing interests}

The authors declare that the research was conducted in the absence of any commercial or financial relationships that could be construed as a potential conflict of interest.
Received: 23 September 2021 Accepted: 18 November 2021

Published online: 01 December 2021

\section{References}

1. Guo Q, Wang Y, Xu D, Nossent J, Pavlos NJ, Xu J. Rheumatoid arthritis: pathological mechanisms and modern pharmacologic therapies. Bone Res. 2018:6:1-14.

2. Almutairi K, Nossent J, Preen D, Keen H, Inderjeeth C. The global prevalence of rheumatoid arthritis: a meta-analysis based on a systematic review. Rheumatol Int. 2021;41:863-77.

3. Scherer HU, Häupl T, Burmester GR. The etiology of rheumatoid arthritis. J Autoimmun. 2020;110:102400.

4. Kobelt $G$, Lindgren $P$, Lindroth $Y$, Jacobson L, Eberhardt K. Modelling the effect of function and disease activity on costs and quality of life in rheumatoid arthritis. Rheumatology. 2005;44:1169-75.

5. Drosos AA, Pelechas E, Kaltsonoudis E, Voulgari PV. Therapeutic options and cost-effectiveness for rheumatoid arthritis treatment. Curr Rheumatol Rep. 2020;22:1-6.

6. Deane KD, Demoruelle MK, Kelmenson LB, Kuhn KA, Norris JM, Holers VM Genetic and environmental risk factors for rheumatoid arthritis. Best Pract Res Clin Rheumatol. 2017;31:3-18.

7. Frisell T, Saevarsdottir S, Askling J. Family history of rheumatoid arthritis: an old concept with new developments. Nat Rev Rheumatol. 2016;12:335-43.

8. Hedström AK, Stawiarz L, Klareskog L, Alfredsson L. Smoking and susceptibility to rheumatoid arthritis in a Swedish population-based case-control study. Eur J Epidemiol. 2018;33:415-23.

9. Murphy D, Bellis K, Hutchinson D. Vapour, gas, dust and fume occupational exposures in male patients with rheumatoid arthritis resident in Cornwall (UK) and their association with rheumatoid factor and anti-cyclic protein antibodies: a retrospective clinical study. BMJ Open. 2018;8:e021754.

10. Scher JU, Abramson SB. The microbiome and rheumatoid arthritis. Nat Rev Rheumatol. 2011;7:569-78.

11. Gómez-Bañuelos E, Mukherjee A, Darrah E, Andrade F. Rheumatoid arthritis-associated mechanisms of Porphyromonas gingivalis and Aggregatibacter actinomycetemcomitans. J Clin Med. 2019:8:1309.

12. Skoczyńska M, Świerkot J. The role of diet in rheumatoid arthritis. Reumatologia. 2018;56:259.

13. Savage E, McCormick D, McDonald S, Moore O, Stevenson M, Cairns A. Does rheumatoid arthritis disease activity correlate with weather conditions? Rheumatol Int. 2015:35:887-90.

14. Smedslund G, Hagen KB. Does rain really cause pain? A systematic review of the associations between weather factors and severity of pain in people with rheumatoid arthritis. Eur J Pain. 2011;15:5-10.

15. Myllykangas-Luosujarvi R, Seuri M, Husman T, Korhonen R, Pakkala K, Aho K. A cluster of inflammatory rheumatic diseases in a moisture-damaged office. Clin Exp Rheumatol. 2002;20:833-6.

16. Luosujärvi R, Husman T, Seuri M, Pietikäinen M, Pollari P, Pelkonen J, et al. Joint symptoms and diseases associated with moisture damage in a health center. Clin Rheumatol. 2003:22:381-5.

17. Engebretsen K, Johansen J, Kezic S, Linneberg A, Thyssen J. The effect of environmental humidity and temperature on skin barrier function and dermatitis. J Eur Acad Dermatol Venereol. 2016;30:223-49.

18. Simmering JE, Polgreen LA, Hornick DB, Sewell DK, Polgreen PM. Weather-dependent risk for Legionnaires' disease, United States. Emerg InfectDis. 2017;23:1843.

19. Zheng B, Hu L, Song X, Wu Z, Cai R, He L, et al. Analgesic effect of different moxibustion durations in rheumatoid arthritis rats. J Tradit Chin Med. 2014;34:90-5.

20. Katagiri C, Sato J, Nomura J, Denda M. Changes in environmental humidity affect the water-holding property of the stratum corneum and its free amino acid content, and the expression of filaggrin in the epidermis of hairless mice. J Dermatol Sci. 2003;31:29-35.

21. Khachigian LM. Collagen antibody-induced arthritis. Nat Protoc. 2006;1:2512-6.

22. Moore AR, Allden S, Bourne T, Denis MC, Kranidioti K, Okoye R, et al. Collagen II antibody-induced arthritis in Tg1278TNFko mice: optimization of 
a novel model to assess treatments targeting human TNFa in rheumatoid arthritis. J Transl Med. 2014;12:1-10.

23. Zhou J, Chen J, Hu C, Xie Z, Li H, Wei S, et al. Exploration of the serum metabolite signature in patients with rheumatoid arthritis using gas chromatography-mass spectrometry. J Pharm Biomed Anal. 2016;127:60-7.

24. He Z, Wang M, Li H, Wen C. GC-MS-based fecal metabolomics reveals gender-attributed fecal signatures in ankylosing spondylitis. Sci Rep. 2019;9:1-9.

25. Edwards C, Cooper C. Early environmental factors and rheumatoid arthritis. Clin Exp Immunol. 2006;143:1-5.

26. Derby MM. Effects of low humidity on health, comfort \& IEQ. ASHRAE J. 2017;59:44.

27. Davis RE, McGregor GR, Enfield KB. Humidity: A review and primer on atmospheric moisture and human health. Environ Res. 2016;144:106-16.

28. Zeng J, Zhang X, Yang J, Bao J, Xiang H, Dear K, et al. Humidity may modify the relationship between temperature and cardiovascular mortality in Zhejiang Province, China. Int J Environ Res Public Health. 2017;14:1383.

29. Tseng C-M, Chen Y-T, Ou S-M, Hsiao Y-H, Li S-Y, Wang S-J, et al. The effect of cold temperature on increased exacerbation of chronic obstructive pulmonary disease: a nationwide study. PLoS One. 2013:8:e57066

30. Toumi A, Chlif S, Bettaieb J, Alaya NB, Boukthir A, Ahmadi ZE, et al. Temporal dynamics and impact of climate factors on the incidence of zoonotic cutaneous leishmaniasis in central Tunisia. PLoS Negl Trop Dis. 2012;6:e1633.

31. Patberg WR, Rasker JJ. Weather effects in rheumatoid arthritis: from controversy to consensus. A review. J Rheumatol. 2004;31:1327-34.

32. Drane D, Berry G, Bieri D, McFarlane A, Brooks P. The association between external weather conditions and pain and stiffness in women with rheumatoid arthritis. J Rheumatol. 1997:24:1309-16.

33. Patberg WR. Correlation of erythrocyte sedimentation rate and outdoor temperature in a patient with rheumatoid arthritis. J Rheumatol. 1997;24:1017.

34. Heaney LM, Deighton K, Suzuki T. Non-targeted metabolomics in sport and exercise science. J Sports Sci. 2019;37:959-67.

35. Kaivosoja SM, Mattila PT, Knuuttila ML. Dietary xylitol protects against the imbalance in bone metabolism during the early phase of collagen type II-induced arthritis in dark agouti rats. Metabolism. 2008;57:1052-5.

36. Zhang Q, Li X, Yin X, Wang H, Fu C, Wang H, et al. Metabolomic profiling reveals serum L-pyroglutamic acid as a potential diagnostic biomarker for systemic lupus erythematosus. Rheumatology. 2021;60:598-606.

37. Qi S, Xu D, Li Q, Xie N, Xia J, Huo Q, et al. Metabonomics screening of serum identifies pyroglutamate as a diagnostic biomarker for nonalcoholic steatohepatitis. Clin Chim Acta. 2017:473:89-95.

38. Hložek T, Kř́ržek T, Tůma P, Bursová M, Coufal P, Čabala R. Quantification of paracetamol and 5-oxoproline in serum by capillary electrophoresis: Implication for clinical toxicology. J Pharm Biomed Anal. 2017; 145:616-20.

\section{Publisher's Note}

Springer Nature remains neutral with regard to jurisdictional claims in published maps and institutional affiliations.

Ready to submit your research? Choose BMC and benefit from:

- fast, convenient online submission

- thorough peer review by experienced researchers in your field

- rapid publication on acceptance

- support for research data, including large and complex data types

- gold Open Access which fosters wider collaboration and increased citations

- maximum visibility for your research: over $100 \mathrm{M}$ website views per year

At BMC, research is always in progress.

Learn more biomedcentral.com/submissions 